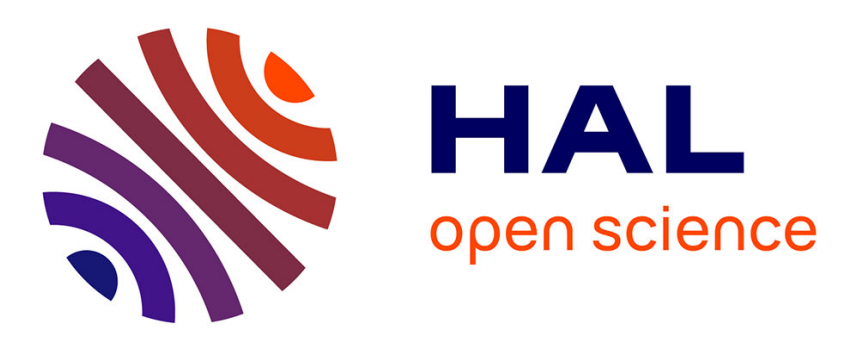

\title{
Maximum Level and Hitting Probabilities in Stochastic Fluid Flows using Matrix Differential Riccati Equations
}

Bruno Sericola, Marie-Ange Remiche

\section{To cite this version:}

Bruno Sericola, Marie-Ange Remiche. Maximum Level and Hitting Probabilities in Stochastic Fluid Flows using Matrix Differential Riccati Equations. [Research Report] PI 1832, 2007, pp.22. inria00123848v2

\section{HAL Id: inria-00123848 \\ https://hal.inria.fr/inria-00123848v2}

Submitted on 11 Jan 2007

HAL is a multi-disciplinary open access archive for the deposit and dissemination of scientific research documents, whether they are published or not. The documents may come from teaching and research institutions in France or abroad, or from public or private research centers.
L'archive ouverte pluridisciplinaire HAL, est destinée au dépôt et à la diffusion de documents scientifiques de niveau recherche, publiés ou non, émanant des établissements d'enseignement et de recherche français ou étrangers, des laboratoires publics ou privés. 


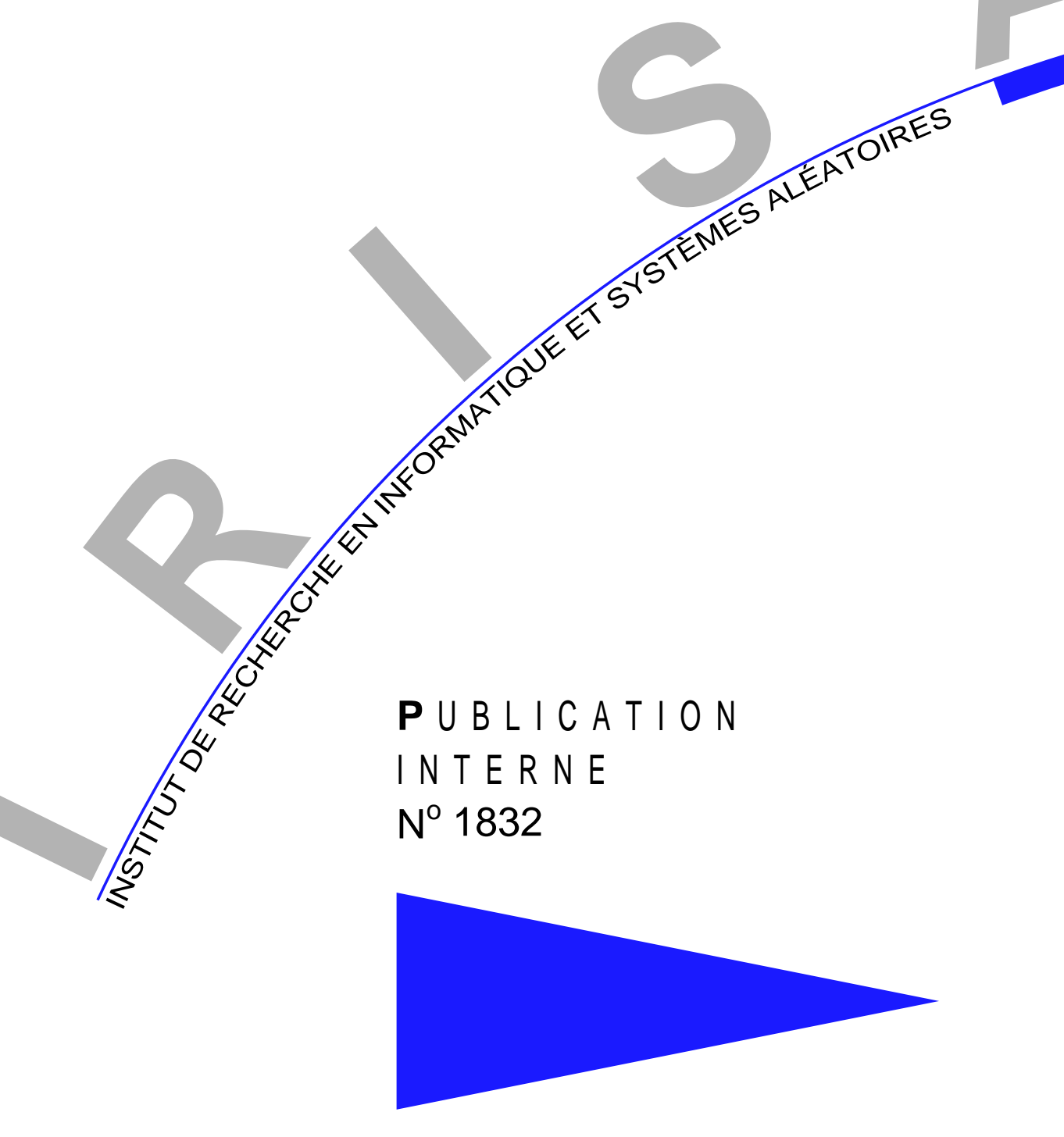

MAXIMUM LEVEL AND HITTING PROBABILITIES IN STOCHASTIC FLUID FLOWS USING MATRIX DIFFERENTIAL RICCATI EQUATIONS BRUNO SERICOLA AND MARIE-ANGE REMICHE 



\title{
Maximum Level and Hitting Probabilities in Stochastic Fluid Flows using Matrix Differential Riccati Equations
}

\author{
Bruno Sericola $^{*}$ and Marie-Ange Remiche ${ }^{* *}$ \\ Systèmes communicants \\ Projet Armor
}

Publication interne $\mathrm{n}^{\circ} 1832$ - Jaunuary 2007 - 22 pages

\begin{abstract}
In this work, we expose a clear methodology to analyze maximum level and hitting probabilities in a Markov driven fluid queue for various initial condition scenarios and in both cases of infinite and finite buffers. Step by step we build up our argument that finally leads to matrix differential Riccati equations for which there exists a unique solution. The power of the methodology resides in the simple probabilistic argument used that permits to obtain analytic solutions of these differential equations. We illustrate our results by a comprehensive fluid model that we exactly solve.
\end{abstract}

Key-words: Fluid Queues, Matrix Differential Riccati Equations, Markov Chains

* IRISA/INRIA, Campus de Beaulieu, 35042 Rennes Cedex, France. Email : bruno.sericola@irisa.fr

** Faculté des Sciences Appliquées, Universit Libre de Bruxelles, Campus du Solbosch, CP 165/15, Avenue F.

D. Roosevelt 50, 1050 Bruxelles, Belgium. Email : mremiche@ulb.ac.be

$9 \%$

Centre National de la Recherche Scientifique (UMR 6074) Université de Rennes 1 - Insa de Rennes
Institut National de Recherche en Informatique et en Automatique - unité de recherche de Rennes 


\section{Niveau maximum et probabilités d'atteinte dans les écoulements stochastiques fluides en utilisant les équations différentielles matricielles de Riccati}

Résumé : On présente, dans cet article, une méthode claire pour analyser le niveau maximum et des probabilités d'atteinte dans une file d'attente fluide en environnement markovien, pour diverses conditions initiales et dans les deux cas de capacités infinie et finie. Pas à pas on construit notre raisonnement qui aboutit finalement à des équations différentielles matricielles de Riccati pour lesquelles il existe une solution unique. La puissance de cette méthode réside dans l'argument probabiliste simple utilisé qui permet d'obtenir des solutions analytiques de ces équations différentielles. Nous illustrons nos résultats par un modèle fluide détaillé que l'on résoud de manière exacte.

Mots clés : Files d'attente fluides, équations différentielles matricielles de Riccati, chaînes de Markov 


\section{Introduction}

In the performance evaluation of packet telecommunication networks, fluid flow models prove very useful to analyze complex systems. Among fluid flow models, fluid queues with Markov modulated input rates play a key role in the recent developments of both queueing theory and performance evaluation of packet networks. The first studies of such queueing systems can be dated back to the works of [12] and [2], who analyzed in the early 1980's fluid models in connection with statistical multiplexing of several identical exponential on-off input sources in a buffer.

The above studies mainly focused on the analysis of the stationary regime and have given rise to a series of theoretical developments. For instance, the work [13] generalizes this model by considering multiple types of exponential on-off inputs and outputs. Using the Wiener-Hopf factorization of finite Markov chains, it has been shown in [16] that the distribution of the buffer level has a matrix exponential form. [14], [8] and [9] respectively exhibit and exploit the similarity between stationary fluid queues in a finite Markovian environment and quasi-birthand-death processes. In [1], a direct connection by stochastic coupling is established between fluid queues and quasi-birth-and-death processes.

Most of the above cited studies have been carried out for finite modulating Markov chains. The analysis of a fluid queue driven by infinite state space Markov chains has also been addressed in many research papers. In [10], the authors used fluid models for a transient analysis of statistical multiplexing on an ATM link. For instance, an analytic expression for the joint stationary distribution of the buffer level and the state of the $M / M / 1$ queue has been obtained in [5]. Queueing systems with more general modulating Markov chains have been studied by several authors. In [20] a fluid queue fed by an infinite general birth and death process is analyzed using spectral theory. In [19], the author considers a finite buffer fluid queue driven by a general Markovian queue with the hypothesis that only one state has a negative drift. A more general approach has been proposed more recently in [11] by using the results of [18].

Various papers, see for instance [7] and [4], have analyzed first passage time probabilities for Markov driven fluid processes. One key parameter in their analysis was the matrix $\Psi$ characterizing the distribution of the phase visited at the end of a busy period in an infinite buffer or finite buffer case Markov fluid queue. We propose in this paper to identify and to analyze several related performance measures to that busy period in order to characterize the maximum and minimum workload that has to be managed by the queue in such a system.

The methodology we use in this work, makes no use of the so-called Laplace-Stieltjes transform as in [4] and [15], but rather exploit another probabilistic argument to directly derive measures of interest. Moreover, obtained equations can be re-interpreted as matrix differential equations that exhibit the so-called Riccati format and for which there exists a unique and explicit solution. We consider both finite and infinite buffer cases for various initial scenarios. The paper is organized as follows.

Section 2 clearly identifies the model we work with and some of the key performance measures of interest in the sequel. We derive in Section 3 an expression for the distribution of the minimum level visited in a busy period for an infinite buffer size fluid queue. Lazily speaking, our approach is based on conditioning on the number of peaks observed in the busy period. This approach also allows us to give another probabilistic interpretation for the matrix $\Psi$ as defined by Ramaswami [14], that is, as previously mentioned, the probability of the visited phase at first return to initial level. Next we extend our analysis to first the joint distribution of both the minimum and maximum level visited in such a busy period and secondly, to the

PI $n^{\circ} 1832$ 
distribution of the maximum level visited. Our approach naturally leads to a Riccati differential system of equations for which there exists an explicit solution. In Section 5, we pursue our analysis by considering the distribution of the maximum trough reached before increasing back to the initial level, instead of decreasing back. A level-reversed argument leads to the analysis of the finite buffer case for the distribution of minimum level ever visited before going back to the initial level. Throughout the whole paper, we clearly identify the relations that link the different performance measures. Finally, we illustrate the efficiency of the method by considering a simple and rather formal example but that clearly shows the power of the method defined in this work.

\section{The model}

We consider a classical fluid queue with an infinite buffer and in which the input and service rates are controlled by a finite homogeneous Markov process $\varphi=\left\{\varphi_{t}, t \geq 0\right\}$ on the finite state space $S$ with infinitesimal generator $T$. The process $\varphi$ is also called the phase process and we denote by $X_{t}$ the amount of fluid in the buffer at time $t$. It is well-known that the pair $\left(\varphi_{t}, X_{t}\right)$ forms a Markov process having a pair of discrete and continuous states. Let $\rho_{i}$ be the input rate and $\eta_{i}$ be the service rate when the Markov process $\varphi$ is in state $i$. We denote by $r_{i}$ the effective input rate of state $i$, that is $r_{i}=\rho_{i}-\eta_{i}$ and we define the diagonal matrix of effective input rates $R=\operatorname{diag}\left(r_{i}, i \in S\right)$. We denote by $S^{0}, S^{-}$and $S^{+}$the subsets of states $i \in S$ such that $r_{i}=0$, $r_{i}<0$ and $r_{i}>0$, respectively. In the same way, we denote by $R_{0}, R_{-}$and $R_{+}$the diagonal matrices $R_{0}=\operatorname{diag}\left(r_{i}, i \in S^{0}\right), R_{-}=\operatorname{diag}\left(-r_{i}, i \in S^{-}\right)$and $R_{+}=\operatorname{diag}\left(r_{i}, i \in S^{+}\right)$. We clearly have $R_{0}=0$, the null matrix. The number of states in $S^{0}, S^{+}$and $S^{-}$are denoted by $n_{0}, n_{-}$ and $n_{+}$, respectively. We partition matrix $T$ in a manner conformant to that decomposition of $S$, by writing

$$
T=\left(\begin{array}{ccc}
T_{00} & T_{0-} & T_{0+} \\
T_{-0} & T_{--} & T_{-+} \\
T_{+0} & T_{+-} & T_{++}
\end{array}\right) .
$$

The Markov process $\varphi$ is supposed to be irreducible and we denoted by $\pi$ its stationary distribution. We thus have $\pi T=0$ and $\pi \mathbb{1}=1$, where $\mathbb{1}$ is the column vector with all its entries equal to 1, its dimension being specified by the context of its use.

We suppose that the stability condition for the fluid queue is satisfied, which means that

$$
\sum_{i \in S} r_{i} \pi_{i}<0
$$

This condition assures that all the busy periods are of finite length a.s. and that the maximum level of the queue during every busy period is finite a.s.

We consider a busy period of that fluid queue and we are interested in several distributions of the fluid queue during a busy period. We denote by $M$ the maximum level in the queue during a busy period. We denote by $H$ the fluid level at the minimum trough and by $L$ its position among all the troughs of that busy period. For any $x \geq 0$, let $\theta(x)$ denote the time greater than $x$ at which the fluid level is equal to $x$, i.e.

$$
\theta(x)=\inf \left\{t>x \mid X_{t}=x\right\}
$$

When $X_{0}=0$, the instants 0 and $\theta(0)$ are respectively the initial and the final instants of the busy period. These variables are shown in Figure 1 in the case where the effective input rates 
are either equal to -1 or equal to 1 . We have numbered the successive peaks and troughs arising during a busy period.

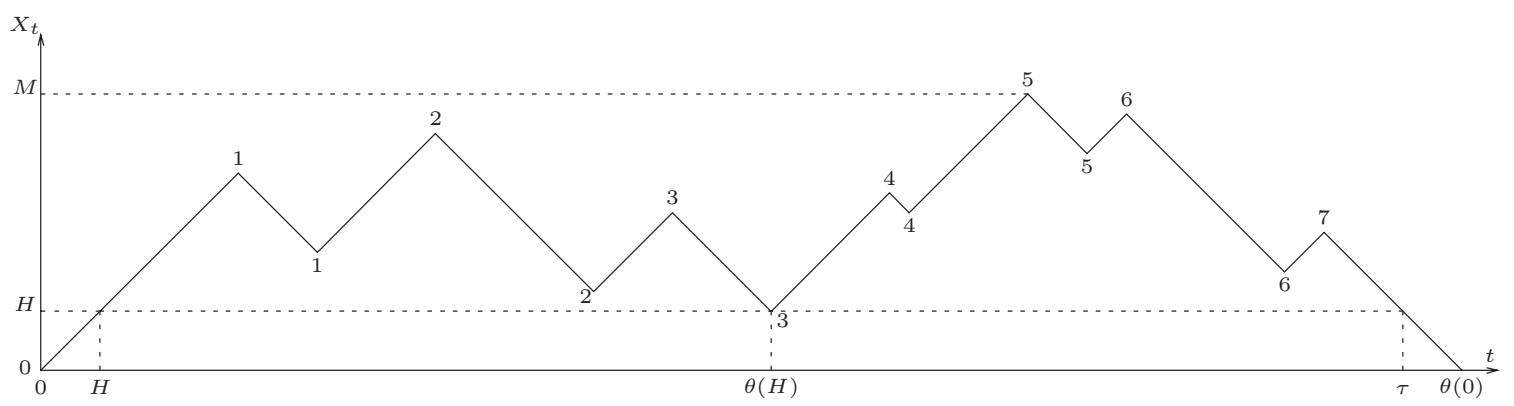

Figure 1: A busy period.

For every $n \geq 2, \ell=1, \ldots, n-1$ and $x \geq 0$, we consider the $n_{+} \times n_{-}$matrix $F_{i, j}(n, \ell, x)$ whose entries are defined, for $i \in S^{+}$and $j \in S^{-}$, by

$$
F_{i, j}(n, \ell, x)=\operatorname{Pr}\left\{\varphi(\theta(0))=j, N=n, L=\ell, H \leq x \mid \varphi(0)=i, X_{0}=0\right\},
$$

where $N$ is the number of peaks arising during a busy period and $L$ is the position of the minimum trough. In the case $N=1$, there is only one peak during the busy period and so, since there is no trough, we do not define the matrix for $n=1$. In the path described in Figure 1 , we have $N=7$ and $L=3$.

We also consider the $n_{+} \times n_{-}$matrix $\Psi_{i, j}(x, y)$ whose entries are defined, for $i \in S^{+}, j \in S^{-}$, $x \geq 0$ and $0 \leq y \leq x$, by

$$
\Psi_{i, j}(x, y)=\operatorname{Pr}\left\{\varphi(\theta(0))=j, M \leq x, H \leq y \mid \varphi(0)=i, X_{0}=0\right\},
$$

and the $n_{-} \times n_{+}$matrix $\Theta_{i, j}(x)$ whose entries are defined, for $i \in S^{-}, j \in S^{+}$and $x>0$, by

$$
\Theta_{i, j}(x)=\operatorname{Pr}\left\{\varphi(\gamma(x))=j \mid \varphi(0)=i, X_{0}=x\right\},
$$

where, for any $x \geq 0, \gamma(x)$ denotes the first positive instant at which the fluid level is equal $x$, i.e.

$$
\gamma(x)=\inf \left\{t>0 \mid X_{t}=x\right\}
$$

We also consider the finite buffer case.

Let us introduce the $\left(n_{-}+n_{+}\right) \times\left(n_{-}+n_{+}\right)$matrix

$$
Q=\left(\begin{array}{ll}
Q_{--} & Q_{-+} \\
Q_{+-} & Q_{++}
\end{array}\right),
$$

where

$$
\begin{aligned}
& Q_{--}=R_{-}^{-1}\left(T_{--}-T_{-0} T_{00}^{-1} T_{0-}\right) \\
& Q_{-+}=R_{-}^{-1}\left(T_{-+}-T_{-0} T_{00}^{-1} T_{0+}\right) \\
& Q_{+-}=R_{+}^{-1}\left(T_{+-}-T_{+0} T_{00}^{-1} T_{0-}\right) \\
& Q_{++}=R_{+}^{-1}\left(T_{++}-T_{+0} T_{00}^{-1} T_{0+}\right)
\end{aligned}
$$


These matrices allow us to restrict our problem to a Markov chain with state space $S^{-} \cup S^{+}$with infinitesimal generator $Q$ and effective input rates equal to -1 or +1 . For instance the matrix $Q_{--}$governs the transitions from $i \in S^{-}$to $j \in S^{-}$without any visit to $S^{+}$. More formally, as shown in [17], $e^{Q_{--} x}(i, j)$ is the probability, starting from state $i$, to reach state $j$ with an accumulated reward equal to $x$ and without leaving the set $S^{0} \cup S^{-}$. Here the accumulated reward corresponds to the amount of fluid generated from the effective input rates of the matrix $R_{-}$. A symmetric interpretation holds for matrix $Q_{++}$. Concerning the matrix $Q_{-+}$, the entries $Q_{-+}(i, j)$ is the rate, rescaled according to matrix $R_{-}$, at which state $j$ is reached from state $i$ either directly from state $i$ or after some time spent in subset $S^{0}$. A symmetric interpretation holds for matrix $Q_{+-}$.

Since we are concerned by quantities such as the minimum trough, the maximum level and hitting probabilities, the fluid queue with parameters $(T, R)$ is equivalent to the fluid queue with parameters $(Q, C)$ where $C$ is the $\left(n_{-}+n_{+}\right) \times\left(n_{-}+n_{+}\right)$matrix

$$
C=\left(\begin{array}{cc}
-I & 0 \\
0 & I
\end{array}\right)
$$

where $I$ is the identity matrix whose dimension is specified by the context of its use. This transformation has been also suggested in [3] and [16].

Thus, in the following, we will consider a fluid queue driven by a Markov chain $\varphi=\left\{\varphi_{t}, t \geq\right.$ $0\}$ with state space $S=S^{-} \cup S^{+}$, infinitesimal generator $Q$ and effective input rates given by matrix $C$, i.e. equal to -1 or equal 1 .

\section{Minimum Trough}

For $i \in S^{+}, j \in S^{-}, n \geq 2, \ell=1, \ldots, n-1$ and $y \geq 0$, we denote by $f_{i, j}(n, \ell, y)$ the joint density associated with the distribution

$$
F_{i, j}(n, \ell, y)=\operatorname{Pr}\left\{\varphi(\theta(0))=j, N=n, L=\ell, H \leq y \mid \varphi(0)=i, X_{0}=0\right\}
$$

and defined by

$$
f_{i, j}(n, \ell, y)=\frac{d F_{i, j}(n, \ell, y)}{d y}
$$

We also introduce, for $n \geq 1$, the $n_{+} \times n_{-}$matrix $W(n)$ whose entries are defined, for $i \in S^{+}$ and $j \in S^{-}$, by

$$
W_{i, j}(n)=\operatorname{Pr}\left\{\varphi(\theta(0))=j, N=n \mid \varphi(0)=i, X_{0}=0\right\} .
$$

We thus have, for $n \geq 2$,

$$
W_{i, j}(n)=\int_{0}^{\infty} \sum_{\ell=1}^{n-1} f_{i, j}(n, \ell, y) d y
$$

The $n_{+} \times n_{-}$matrix containing the terms $f_{i, j}(n, \ell, y)$ is denoted by $f(n, \ell, y)$ and is given together with matrix $W(1)$ by the following theorem.

Theorem 3.1. For $n \geq 2, \ell=1, \ldots, n-1$ and $y \geq 0$, we have

$$
W(1)=\int_{0}^{\infty} e^{Q_{++} y} Q_{+-} e^{Q_{--} y} d y
$$

and

$$
f(n, \ell, y)=e^{Q_{++} y} W(\ell) Q_{-+} W(n-\ell) e^{Q_{--} y} .
$$


Proof. The proof follows the same arguments developed in [7]. To obtain the expression for $W(1)$ we consider a sample path starting from fluid level 0 in phase $i \in S^{+}$and returning to level 0 in phase $j \in S^{-}$with only one peak of height $y$ in between. Such a sample path can be broken up into three stages which are :

1. The phase process $\varphi$ starts in phase $i \in S^{+}$and reaches some state $k \in S^{+}$at time $y$ without leaving subset $S^{+}$. This means that the fluid level increases from level 0 to level $y$. As seen in the previous section, the corresponding probability is equal to $e^{Q_{++} y}(i, k)$.

2. Since a peak (of height equal to $y$ ) occurs, this means that a transition of the phase process $\varphi$ occurs from state $k$ to some state $h \in S^{-}$. The corresponding transition rate is equal to $Q_{+-}(k, h)$.

3. Starting from state $h \in S^{-}$, the process $\varphi$ reaches state $j \in S^{-}$at time $y$ without leaving subset $S^{-}$. This means that the fluid level decreases from level $y$ to level 0 . As seen in the previous section, the corresponding probability is equal to $e^{Q_{--}}(h, j)$.

We thus obtain

$$
W_{i, j}(1)=\int_{0}^{\infty} \sum_{k \in S^{+}} \sum_{h \in S^{-}} e^{Q_{++} y}(i, k) Q_{+-}(k, h) e^{Q_{--} y}(h, j) d y
$$

that is

$$
W(1)=\int_{0}^{\infty} e^{Q_{++}} Q_{+-} e^{Q_{--} y} d y .
$$

Let now $n \geq 2$ and $1 \leq \ell \leq n-1$. A typical example of such a sample path is shown in Figure 1. It is a path starting from fluid level 0 in phase $i \in S^{+}$and returning to level 0 in phase $j \in S^{-}$with $N=n$ peaks, with the minimum trough of height $H=y$ at position $L=\ell$. Such a sample path can be broken up into five stages which are :

1. As we observe, $H$ denotes the minimum level as well as the amount of time needed to reach it. The phase process $\varphi$ starts in phase $i \in S^{+}$and reaches some state $k \in S^{+}$at time $H=y$ without leaving subset $S^{+}$. This means that the fluid level increases from level 0 to level $y$. As seen in the previous section, the corresponding probability is equal to $e^{Q_{++} y}(i, k)$.

2. Starting from phase $k \in S^{+}$and level $y$, the fluid process returns for the first time to level $y$ in some phase $h \in S^{-}$. By definition of $H$ and $L$, the number of peaks between instants $H$ and $\theta(H)$ is equal to $\ell$. By the spatial homogeneity of the process, the corresponding probability is equal to $W_{k, h}(\ell)$.

3. Since a trough (of height equal to $y$ at time $\theta(H)$ ) occurs, this means that a transition of the phase process $\varphi$ occurs from state $h \in S^{-}$to some state $m \in S^{+}$. The corresponding transition rate is equal to $Q_{-+}(h, m)$.

4. Once again, starting from phase $m \in S^{+}$and level $y$, the fluid process returns for the first time to level $y$ in some phase $v \in S^{-}$. By definition of $H$ and $L$, the number of peaks between instants $\theta(H)$ and $\tau=\theta(0)-H$ is equal to $n-\ell$. By the spatial homogeneity of the process, the corresponding probability is equal to $W_{m, v}(n-\ell)$. 
5. The phase process $\varphi$ starts in phase $v \in S^{-}$and level $y$. It reaches level 0 in state $j$ without leaving subset $S^{-}$, at time $\varphi(\theta(0))$. This means that the fluid level decreases from level $y$ to level 0 . As seen in the previous section, the corresponding probability is equal to $e^{Q_{--y}}(v, j)$.

We thus obtain

$$
f_{i, j}(n, \ell, y)=\sum_{k \in S^{+}} \sum_{h \in S^{-}} \sum_{m \in S^{+}} \sum_{v \in S^{-}} e^{Q_{++} y}(i, k) W_{k, h}(\ell) Q_{-+}(h, m) W_{m, v}(n-\ell) e^{Q_{--} y}(v, j),
$$

that is

$$
f(n, \ell, y)=e^{Q_{++} y} W(\ell) Q_{-+} W(n-\ell) e^{Q_{--} y} .
$$

The distribution of the number of peaks during a busy period is given in the following corollary.

Corollary 3.2. For every $n \geq 2$, we have

$$
W(n)=\int_{0}^{\infty} e^{Q_{++} y} \sum_{\ell=1}^{n-1} W(\ell) Q_{-+} W(n-\ell) e^{Q_{--} y} d y .
$$

Proof. It suffices to write

$$
W(n)=\int_{0}^{\infty} \sum_{\ell=1}^{n-1} f_{i, j}(n, \ell, y) d y
$$

to get the result.

Let $\Psi$ denote $n_{+} \times n_{-}$the matrix whose entries are defined, for $i \in S^{+}$and $j \in S^{-}$, by

$$
\Psi_{i, j}=\operatorname{Pr}\left\{\varphi(\theta(0))=j \mid \varphi(0)=i, X_{0}=0\right\} .
$$

$\Psi_{i, j}$ is the probability that the phase at the instant of the first return to the initial level is equal to $j$. By spatial homogeneity, this probability is independent of the value of the initial level. We have

$$
\Psi=\sum_{n=1}^{\infty} W(n) .
$$

In the next theorem, we give a relation satisfied by matrix $\Psi$.

\section{Theorem 3.3.}

$$
\Psi=\int_{0}^{\infty} e^{Q_{++} y} Q_{+-} e^{Q_{--} y} d y+\int_{0}^{\infty} e^{Q_{++} y} \Psi Q_{-+} \Psi e^{Q_{--} y} d y
$$

Proof. From Corollary 3.2, we have, for $n \geq 2$,

$$
W(n)=\int_{0}^{\infty} e^{Q_{++} y} \sum_{\ell=1}^{n-1} W(\ell) Q_{-+} W(n-\ell) e^{Q_{--} y} d y .
$$


By summation over $n$, we get, using the monotone convergence theorem,

$$
\begin{aligned}
\Psi & =W(1)+\sum_{n=2}^{\infty} \int_{0}^{\infty} e^{Q_{++} y} \sum_{\ell=1}^{n-1} W(\ell) Q_{-+} W(n-\ell) e^{Q_{--} y} d y \\
& =W(1)+\int_{0}^{\infty} e^{Q_{++} y} \sum_{n=2}^{\infty} \sum_{\ell=1}^{n-1} W(\ell) Q_{-+} W(n-\ell) e^{Q_{--} y} d y \\
& =W(1)+\int_{0}^{\infty} e^{Q_{++} y} \sum_{\ell=1}^{\infty} \sum_{n=\ell+1}^{\infty} W(\ell) Q_{-+} W(n-\ell) e^{Q_{--} y} d y \\
& =W(1)+\int_{0}^{\infty} e^{Q_{++} y} \sum_{\ell=1}^{\infty} W(\ell) Q_{-+} \sum_{n=\ell+1}^{\infty} W(n-\ell) e^{Q_{--} y} d y \\
& =W(1)+\int_{0}^{\infty} e^{Q_{++} y} \Psi Q_{-+} \Psi e^{Q_{--} y} d y,
\end{aligned}
$$

which is the desired result.

It is easily checked that matrix $\Psi$ is solution to the following matrix algebraic Riccati equation

$$
Q_{++} \Psi+\Psi Q_{--}+\Psi Q_{-+} \Psi+Q_{+-}=0
$$

This equation has been considered in several papers and several algorithms have been developed to compute $\Psi$ which is the minimal solution of this equation. See for instance [7], [6] and the references therein. by

Let $F(x)$ denote the $n_{+} \times n_{-}$the matrix whose entries are defined, for $i \in S^{+}$and $j \in S^{-}$,

$$
F_{i, j}(x)=\operatorname{Pr}\{\varphi(\theta(0))=j, H \leq x \mid \varphi(0)=i\} .
$$

$F_{i, j}(x)$ is the probability that the phase at the instant of the first return to the initial level is equal to $j$ with a minimum trough of height less than or equal to $x$. This probability is defined only when the minimum trough exists. The minimum trough exists if and only if the number of peaks $N$ is greater than or equal to 2. Moreover, when it exists, we have $H>0$. Thus we define the event $H=0$ to represent the non-existence of the minimum trough, i.e. the case where the number of peaks is equal to 1.

We then have the following result.

Corollary 3.4. For every $x \geq 0$,

$$
F(x)=\int_{0}^{\infty} e^{Q_{++} y} Q_{+-} e^{Q_{--} y} d y+\int_{0}^{x} e^{Q_{++} y} \Psi Q_{-+} \Psi e^{Q_{--} y} d y .
$$

Proof. By definition, we have

$$
\begin{aligned}
F_{i, j}(x) & =\operatorname{Pr}\{\varphi(\theta(0))=j, H=0 \mid \varphi(0)=i\}+\operatorname{Pr}\{\varphi(\theta(0))=j, 0<H \leq x \mid \varphi(0)=i\} \\
& =\operatorname{Pr}\{\varphi(\theta(0))=j, N=1 \mid \varphi(0)=i\}+\operatorname{Pr}\{\varphi(\theta(0))=j, N \geq 2, H \leq x \mid \varphi(0)=i\} \\
& =W_{i, j}(1)+\int_{0}^{x} \sum_{n=2}^{\infty} \sum_{\ell=0}^{n-1} f_{i, j}(n, \ell, y) d y .
\end{aligned}
$$

Following the same lines used in the proof of Theorem 3.3, we get the result. 


\section{$4 \quad$ Maximum level}

We consider in this section the maximum fluid level $M$ reached during a busy period. A typical path is shown in Figure 1. For $i \in S^{+}, j \in S^{-}, x \geq 0$ and $0 \leq y \leq x$, we denote by $\psi_{i, j}(x, y)$ the marginal density associated with the distribution

$$
\Psi_{i, j}(x, y)=\operatorname{Pr}\left\{\varphi(\theta(0))=j, M \leq x, H \leq y \mid \varphi(0)=i, X_{0}=0\right\},
$$

and defined by

$$
\psi_{i, j}(x, y)=\frac{\partial \Psi_{i, j}(x, y)}{\partial y}
$$

For $x \geq 0$, we denote by $\Psi(x)$ the $n_{+} \times n_{-}$matrix containing the $\Psi_{i, j}(x)$ defined, for $i \in S^{+}$ and $j \in S^{-}$, by

$$
\Psi_{i, j}(x)=\operatorname{Pr}\left\{\varphi(\theta(0))=j, M \leq x \mid \varphi(0)=i, X_{0}=0\right\} .
$$

$\Psi_{i, j}(x)$ is the probability that, starting from phase $i \in S^{+}$and level $u \geq 0$, the phase at the instant of the first return to the initial level $u$ is equal to $j$ and the maximum level is less than or equal to $x+u$. By the spatial homogeneity, this probability is independent of $u$. The $n_{+} \times n_{-}$matrix containing the terms $\psi_{i, j}(x, y)$ is denoted by $\psi(x, y)$ and is given by the following theorem.

Theorem 4.1. For $x \geq 0$ and $0 \leq y \leq x$, we have

$$
\Psi(x, 0)=\int_{0}^{x} e^{Q_{++} y} Q_{+-} e^{Q_{--}} d y
$$

and

$$
\psi(x, y)=e^{Q_{++} y} \Psi(x-y) Q_{-+} \Psi(x-y) e^{Q_{--} y} .
$$

Proof. We proceed as for the proof of Theorem 3.1. The term $\Psi(x, 0)$ corresponds to the case where there is only one peak, and thus no trough, which means, as defined in the previous section, that $H=0$. To obtain the expression for $\Psi(x, 0)$ we consider a sample path starting from fluid level 0 in phase $i \in S^{+}$and returning to level 0 in phase $j \in S^{-}$with only one peak of height $y$ (with $y \leq x$ ) in between. Such a sample path can be broken up into three stages which are :

1. The phase process $\varphi$ starts in phase $i \in S^{+}$and reaches some state $k \in S^{+}$at time $y$ without leaving subset $S^{+}$. This means that the fluid level increases from level 0 to level $y$. As seen in the previous section, the corresponding probability is equal to $e^{Q_{++} y}(i, k)$.

2. Since a peak (of height equal to $y$ ) occurs, this means that a transition of the phase process $\varphi$ occurs from state $k$ to some state $h \in S^{-}$. The corresponding transition rate is equal to $Q_{+-}(k, h)$.

3. Starting from state $h \in S^{-}$, the process $\varphi$ reaches state $j \in S^{-}$at time $y$ without leaving subset $S^{-}$. This means that the fluid level decreases from level $y$ to level 0 . As seen in the previous section, the corresponding probability is equal to $e^{Q_{--} y}(h, j)$.

We thus obtain

$$
\Psi_{i, j}(x, 0)=\int_{0}^{x} \sum_{k \in S^{+}} \sum_{h \in S^{-}} e^{Q_{++} y}(i, k) Q_{+-}(k, h) e^{Q_{--} y}(h, j) d y
$$


that is

$$
\Psi(x, 0)=\int_{0}^{x} e^{Q_{++} y} Q_{+-} e^{Q_{--} y} d y .
$$

We consider now the case where a minimum trough exists, i.e. $H>0$. A typical example of such a sample path is shown in Figure 1. It is a path starting from fluid level 0 in phase $i \in S^{+}$and returning to level 0 in phase $j \in S^{-}$with at least two peaks (i.e. $H>0$ ), with the minimum trough and the maximum level less than or equal to $x$. Such a sample path can be broken up into five stages which are :

1. The phase process $\varphi$ starts in phase $i \in S^{+}$and reaches some state $k \in S^{+}$at time $H=y$ (with $y \leq x$ ) without leaving subset $S^{+}$. This means that the fluid level increases from level 0 to level $y$. As seen in the previous section, the corresponding probability is equal to $e^{Q_{++} y}(i, k)$.

2. Starting from phase $k \in S^{+}$and level $y$, the fluid process returns for the first time to level $y$ in some phase $h \in S^{-}$, without exceeding level $x$. By the spatial homogeneity of the process, the corresponding probability is equal to $\Psi(x-y)$.

3. Since a trough (of height equal to $y$ at time $\theta(H)$ ) occurs, this means that a transition of the phase process $\varphi$ occurs from state $h \in S^{-}$to some state $m \in S^{+}$. The corresponding transition rate is equal to $Q_{-+}(h, m)$.

4. Once again, starting from phase $m \in S^{+}$and level $y$, the fluid process returns for the first time to level $y$ in some phase $v \in S^{-}$without exceeding level $x$. By the spatial homogeneity of the process, the corresponding probability is equal to $\Psi(x-y)$.

5. The phase process $\varphi$ starts in phase $v \in S^{-}$and level $y$. It reaches level 0 in state $j$ without leaving subset $S^{-}$, at time $\varphi(\theta(0))$. This means that the fluid level decreases from level $y$ to level 0 . As seen in the previous section, the corresponding probability is equal to $e^{Q_{--y}}(v, j)$.

We thus obtain

$$
\psi_{i, j}(x, y)=\sum_{k \in S^{+}} \sum_{h \in S^{-}} \sum_{m \in S^{+}} \sum_{v \in S^{-}} e^{Q_{++} y}(i, k) \Psi_{k, h}(x-y) Q_{-+}(h, m) \Psi_{m, v}(x-y) e^{Q_{--} y}(v, j),
$$

that is

$$
\psi(x, y)=e^{Q_{++} y} \Psi(x-y) Q_{-+} \Psi(x-y) e^{Q_{--}} .
$$

The matrices $\Psi(x, z)$ and $\Psi(x)$ are given by the following corollary.

Corollary 4.2. For $x \geq 0$ and $0 \leq z \leq x$, we have

$$
\begin{gathered}
\Psi(x, z)=\int_{0}^{x} e^{Q_{++} y} Q_{+-} e^{Q_{--}} d y+\int_{0}^{z} e^{Q_{++} y} \Psi(x-y) Q_{-+} \Psi(x-y) e^{Q_{--} y} d y . \\
\Psi(x)=\int_{0}^{x} e^{Q_{++} y} Q_{+-} e^{Q_{--}} d y+\int_{0}^{x} e^{Q_{++} y} \Psi(x-y) Q_{-+} \Psi(x-y) e^{Q_{--} y} d y .
\end{gathered}
$$


Proof. It suffices to write

$$
\Psi(x, z)=\Psi(x, 0)+\int_{0}^{z} \psi(x, y) d y
$$

and $\Psi(x)=\Psi(x, x)$.

Note that it can be easily checked that $\lim _{x \longrightarrow \infty} \Psi(x, z)=F(z)$ and $\lim _{x \longrightarrow \infty} \Psi(x)=\Psi$.

We denote by $\Psi^{\prime}(x)$ the derivative of $\Psi$ with respect to $x$. We then have the following result.

Theorem 4.3. The function $\Psi(x)$ satisfies the following matrix differential Riccati equation

$$
\Psi^{\prime}(x)=Q_{++} \Psi(x)+\Psi(x) Q_{--}+\Psi(x) Q_{-+} \Psi(x)+Q_{+-},
$$

with $\Psi(0)=0$ as initial condition.

Proof. By definition of function $\Psi(x)$ in relation (1), the initial condition is trivially given by $\Psi(0)=0$, since the maximum level $M$ during a busy period is positive. By using a variable change, relation (3) can be written as

$$
\Psi(x)=\int_{0}^{x} e^{Q_{++} y} Q_{+-} e^{Q_{--} y} d y+e^{Q_{++} x} \int_{0}^{x} e^{-Q_{++} y} \Psi(y) Q_{-+} \Psi(y) e^{-Q_{--} y} d y e^{Q_{--}} .
$$

We introduce the notation

$$
\begin{gathered}
\alpha(x)=\int_{0}^{x} e^{Q_{++} y} Q_{+-} e^{Q_{--} y} d y \\
\beta(x)=\int_{0}^{x} e^{-Q_{++} y} \Psi(y) Q_{-+} \Psi(y) e^{-Q_{--}} d y .
\end{gathered}
$$

It is easy to check that

$$
e^{Q_{++} x} Q_{+-} e^{Q_{--} x}-Q_{++} \alpha(x)-\alpha(x) Q_{--}=Q_{+-} .
$$

By differentiating (3), we get

$$
\begin{aligned}
\Psi^{\prime}(x) & =e^{Q_{++} x} Q_{+-} e^{Q_{--} x}+Q_{++} e^{Q_{++} x} \beta(x) e^{Q_{--} x}+e^{Q_{++} x}\left[\beta^{\prime}(x) e^{Q_{--} x}+\beta(x) e^{Q_{--}} Q_{--}\right] \\
& =e^{Q_{++} x} Q_{+-} e^{Q_{--} x}+Q_{++}[\Psi(x)-\alpha(x)]+e^{Q_{++} x} \beta^{\prime}(x) e^{Q_{--} x}+[\Psi(x)-\alpha(x)] Q_{--} \\
& =Q_{++} \Psi(x)+\Psi(x) Q_{--}+e^{Q_{++} x} \beta^{\prime}(x) e^{Q_{--} x}+Q_{+-} \\
& =Q_{++} \Psi(x)+\Psi(x) Q_{--}+\Psi(x) Q_{-+} \Psi(x)+Q_{+-},
\end{aligned}
$$

which is the desired result.

With the initial condition $\Psi(0)=0$, the Cauchy-Lipschitz theorem assures that the matrix differential Riccati equation (4) has a unique solution. This solution is thus given, for $x \geq 0$, by (1).

By definition of matrix diagonal $C$, we have

$$
C Q=\left(\begin{array}{cc}
-Q_{--} & -Q_{-+} \\
Q_{+-} & Q_{++}
\end{array}\right) .
$$

According to the decomposition $S=S^{-} \cup S^{+}$, we define the four matrices $A(x), B(x), C(x)$ and $D(x)$ occurring in the matrix $e^{C Q x}$ by writing

$$
e^{C Q x}=\left(\begin{array}{cc}
A(x) & B(x) \\
C(x) & D(x)
\end{array}\right)
$$

The following theorem gives an expression of the solution $\Psi(x)$ to the matrix differential Riccati equation (4). 
Theorem 4.4. For every $x \geq 0$, we have

$$
\Psi(x)=C(x) A(x)^{-1} .
$$

Proof. Let us consider the following linear differential equation

$$
Y^{\prime}(x)=\left(-Q_{--}-Q_{-+} \Psi(x)\right) Y(x) \text { and } Y(0)=I .
$$

The function $\Psi$ being continuous, this linear system has a unique solution which is invertible. We now define the $n_{+} \times n_{-}$matrix $Z(x)$ by

$$
Z(x)=\Psi(x) Y(x) .
$$

Using this definition, equation (5) becomes

$$
Y^{\prime}(x)=-Q_{--} Y(x)-Q_{-+} Z(x) .
$$

By differentiating $Z(x)$ with respect to $x$, we obtain from (4) and (6)

$$
\begin{aligned}
Z^{\prime}(x)= & \Psi^{\prime}(x) Y(x)+\Psi(x) Y^{\prime}(x) \\
= & \left(Q_{++} \Psi(x)+\Psi(x) Q_{--}+\Psi(x) Q_{-+} \Psi(x)+Q_{+-}\right) Y(x) \\
& +\Psi(x)\left(-Q_{--} Y(x)-Q_{-+} Z(x)\right) \\
= & Q_{++} Z(x)+\Psi(x) Q_{-+} \Psi(x) Y(x)+Q_{+-} Y(x)-\Psi(x) Q_{-+} Z(x) \\
= & Q_{++} Z(x)+Q_{+-} Y(x) .
\end{aligned}
$$

Putting together equations (6) and (7) we obtain

$$
\left(\begin{array}{c}
Y^{\prime}(x) \\
Z^{\prime}(x)
\end{array}\right)=\left(\begin{array}{cc}
-Q_{--} & -Q_{-+} \\
Q_{+-} & Q_{++}
\end{array}\right)\left(\begin{array}{c}
Y(x) \\
Z(x)
\end{array}\right)=C Q\left(\begin{array}{c}
Y(x) \\
Z(x)
\end{array}\right),
$$

with $Y(0)=I$ and $Z(0)=0$. The solution to that equation is given by

$$
\left(\begin{array}{c}
Y(x) \\
Z(x)
\end{array}\right)=e^{C Q x}\left(\begin{array}{c}
I \\
0
\end{array}\right)=\left(\begin{array}{c}
A(x) \\
C(x)
\end{array}\right)
$$

which means that $Y(x)=A(x), Z(x)=C(x)$ and thus, since $A(x)$ is invertible, we have $\Psi(x)=C(x) A(x)^{-1}$.

\section{Maximum peak}

We consider the first return to the initial level $x$ when $x>0$ and the initial phase is in $S^{-}$. More formally, we introduce the $n_{-} \times n_{+}$matrix $G(x, y)$ whose entries are defined, for $i \in S^{-}$, $j \in S^{+}, x>0$ and $0 \leq y \leq x$, by

$$
G_{i, j}(x, y)=\operatorname{Pr}\left\{\varphi(\gamma(x))=j, M \leq y \mid \varphi(0)=i, X_{0}=x\right\}
$$

where $M$ denotes the maximum peak between instants 0 and $\gamma(x)$. The $n_{-} \times n_{+}$matrix $\Theta(x)$ whose entries are defined, for $i \in S^{-}, j \in S^{+}$and $x>0$, by

PI ${ }^{\circ} 1832$

$$
\Theta_{i, j}(x)=\operatorname{Pr}\left\{\varphi(\gamma(x))=j \mid \varphi(0)=i, X_{0}=x\right\},
$$


satisfies

$$
\Theta(x)=G(x, x)
$$

Remark that, by spatial homogeneity, we have $\operatorname{Pr}\left\{\varphi(\gamma(x))=j \mid \varphi(0)=i, X_{0}=x\right\}=\Psi_{i, j}$, when $i \in S^{+}$and $j \in S^{-}$.

For $i \in S^{-}$and $j \in S^{+}$, the problem is more complicated because of the influence of the boundary level zero which makes the hitting probability $\Theta_{i, j}(x)$ dependent of $x$.

A typical example of such a sample path is shown in Figure 2.
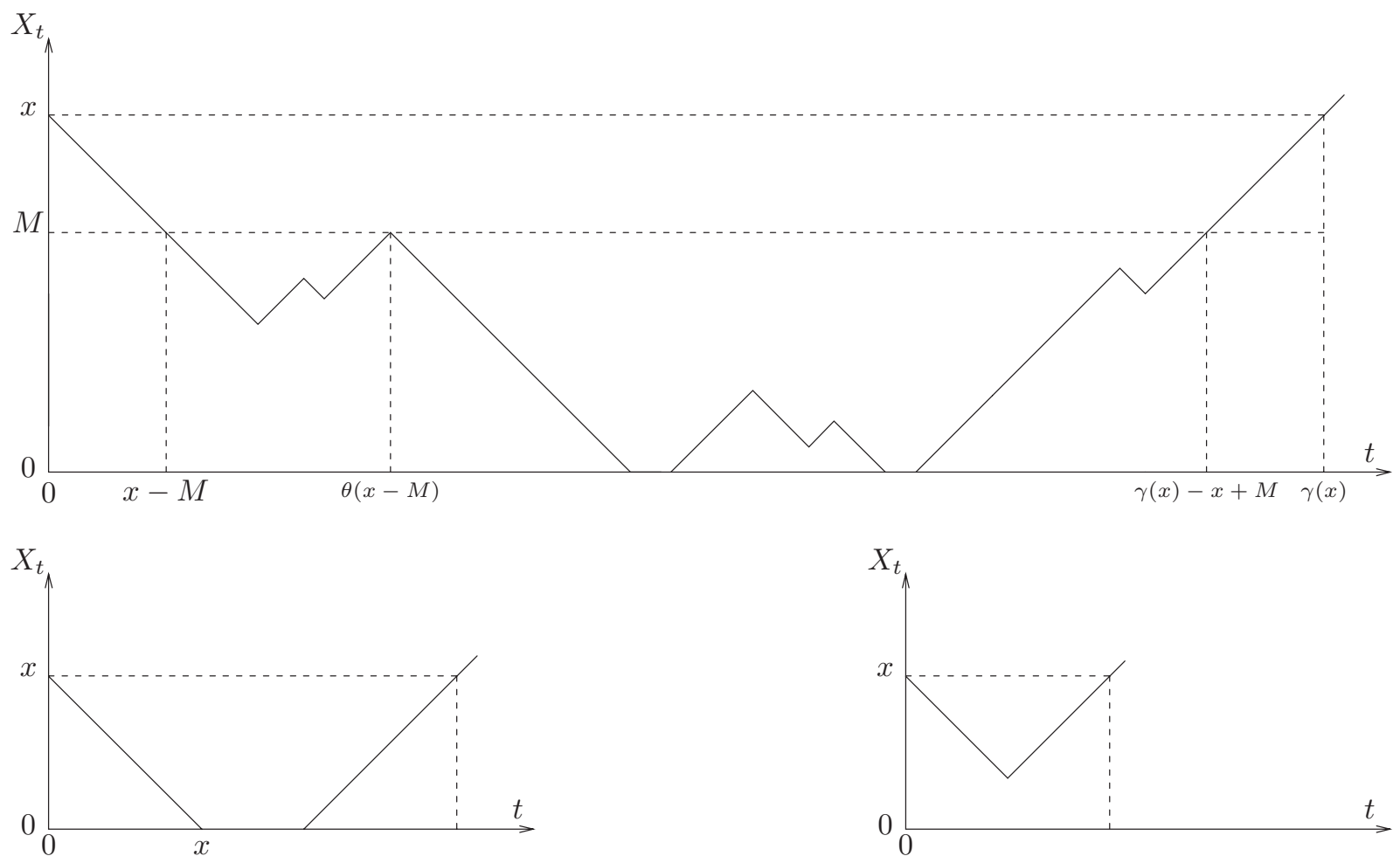

Figure 2: A period of level $<x$.

We denote by $g_{i, j}(x, y)$ the density associated with the distribution $G_{i, j}(x, y)$, i.e.

$$
g_{i, j}(x, y)=\frac{\partial G_{i, j}(x, y)}{\partial y}
$$

Theorem 5.1. For $x>0$, we have

$$
G(x, 0)=\int_{0}^{x} e^{Q_{--}} Q_{-+} e^{Q_{++} y} d y-Q_{--}^{-1} e^{Q_{--}} Q_{-+} e^{Q_{++} x}
$$

and, for $0 \leq y \leq x$,

$$
g(x, y)=e^{Q_{--} y} \Theta(x-y) Q_{+-} \Theta(x-y) e^{Q_{++} y}
$$

Proof. We proceed as for the proof of theorems 3.1 and 4.1. The term $G(x, 0)$ corresponds to the case where there are no peaks in the interval $[0, \gamma(0)]$, i.e. where $M=0$. This situation corresponds to the paths shown in the down part of Figure 2. The left part corresponds to the case the height of the unique trough, denoted by $H$, is zero and the right part corresponds to the case where $H$ is positive. We thus have

$$
\begin{aligned}
G(x, 0)= & \operatorname{Pr}\left\{\varphi(\gamma(x))=j, M=0, H>0 \mid \varphi(0)=i, X_{0}=x\right\} \\
& +\operatorname{Pr}\left\{\varphi(\gamma(x))=j, M=0, H=0 \mid \varphi(0)=i, X_{0}=x\right\} .
\end{aligned}
$$


The first term is the symmetric term of $\Psi(x, 0)$, so we easily get

$$
\operatorname{Pr}\left\{\varphi(\gamma(x))=j, M=0, H>0 \mid \varphi(0)=i, X_{0}=x\right\}=\int_{0}^{x} e^{Q_{--} y} Q_{-+} e^{Q_{++} y} d y .
$$

For the second term, which corresponds to the sample path on the bottom left of Figure 2, the phase process $\varphi$ starts in phase $i \in S^{-}$with a level $x$. It stays in subset $S^{-}$for a duration $y \geq x$, reaching some state $k \in S^{-}$and thus with a level 0 . Next a transition occurs from state $k \in S^{-}$to state $h \in S^{+}$, with rate $Q_{+_{-}}(k, h)$, and the process $\varphi$ reaches state $j \in S^{+}$at time $x$ without leaving subset $S^{+}$. We thus have

$$
\begin{aligned}
\operatorname{Pr}\left\{\varphi(\gamma(x))=j, M=0, H=0 \mid \varphi(0)=i, X_{0}=x\right\} & =\int_{x}^{\infty} e^{Q_{--} y} d y Q_{-+} e^{Q_{++} x} \\
& =-Q_{--}^{-1} e^{Q_{--}} Q_{-+} e^{Q_{++}}
\end{aligned}
$$

We consider now the case where there is at least one peak, i.e. $M>0$. A typical example of such a sample path is shown in the upper of Figure 2. Such a sample path can be broken up into five stages which are :

1. The phase process $\varphi$ starts in phase $i \in S^{-}$and reaches some state $k \in S^{-}$at time $x-M=y$ without leaving subset $S^{-}$. This means that the fluid level decreases from level $x$ to level $x-y$. As seen in the previous section, the corresponding probability is equal to $e^{Q-y}(i, k)$.

2. Starting from phase $k \in S^{-}$and level $x-y$, the fluid process returns for the first time to level $x-y$ in some phase $h \in S^{+}$, without exceeding level $x-y$. By the spatial homogeneity of the process, the corresponding probability is equal to $\Theta(x-y)$.

3. Since a peak (of height equal to $x-y$ at time $\theta(x-H)$ ) occurs, this means that a transition of the phase process $\varphi$ occurs from state $h \in S^{+}$to some state $m \in S^{-}$. The corresponding transition rate is equal to $Q_{-+}(h, m)$.

4. Once again, starting from phase $m \in S^{-}$and level $x-y$, the fluid process returns for the first time to level $x-y$ in some phase $v \in S^{+}$without exceeding level $x-y$. By the spatial homogeneity of the process, the corresponding probability is equal to $\Theta(x-y)$.

5. The phase process $\varphi$ starts in phase $v \in S^{+}$and level $x-y$. It reaches level $x$ in state $j$ without leaving subset $S^{+}$, at time $\varphi(\gamma(x))$. This means that the fluid level increases from level $x-y$ to level $x$. As seen in the previous section, the corresponding probability is equal to $e^{Q_{++} y}(v, j)$.

We thus obtain

$$
g_{i, j}(x, y)=\sum_{k \in S^{-}} \sum_{h \in S^{+}} \sum_{m \in S^{-}} \sum_{v \in S^{+}} e^{Q_{++} y}(i, k) \Theta_{k, h}(x-y) Q_{+-}(h, m) \Theta_{m, v}(x-y) e^{Q_{++} y}(v, j),
$$

that is

$$
g(x, y)=e^{Q_{--} y} \Theta(x-y) Q_{+-} \Theta(x-y) e^{Q_{++} y} .
$$

The matrices $G(x, z)$ and $\Theta(x)$ are given by the following corollary.

PI ${ }^{\circ} 1832$ 
Corollary 5.2. For $x \geq 0$ and $0 \leq z \leq x$, we have

$G(x, z)=\int_{0}^{x} e^{Q_{--}} Q_{-+} e^{Q_{++} y} d y-e^{Q_{--}} Q_{--}^{-1} Q_{-+} e^{Q_{++} x}+\int_{0}^{z} e^{Q_{--}} \Theta(x-y) Q_{+-} \Theta(x-y) e^{Q_{++} y} d y$.

$\Theta(x)=\int_{0}^{x} e^{Q_{--}} Q_{-+} e^{Q_{++} y} d y-e^{Q_{--} x} Q_{--}^{-1} Q_{-+} e^{Q_{++} x}+\int_{0}^{x} e^{Q_{--y}} \Theta(x-y) Q_{+-} \Theta(x-y) e^{Q_{++} y} d y$.

Proof. It suffices to write

$$
G(x, z)=G(x, 0)+\int_{0}^{z} g(x, y) d y
$$

and $\Theta(x)=G(x, x)$.

The following result shows that $\Theta(x)$ satisfies a matrix differential Riccati equation.

Theorem 5.3. $\Theta(0)=-Q_{--}^{-1} Q_{-+}$and, for $x>0$, we have

$$
\Theta^{\prime}(x)=Q_{--} \Theta(x)+\Theta(x) Q_{++}+\Theta(x) Q_{+-} \Theta(x)+Q_{-+}
$$

Proof. The proof is based on equation (11) and is thus quite similar to the proof of Theorem 4.3 .

Again, the Cauchy-Lipschitz theorem assures that the matrix differential Riccati equation (12) with the initial condition $\Theta(0)=-Q_{--}^{-1} Q_{-+}$has a unique solution. The following theorem gives an expression of the solution $\Theta(x)$ to that equation.

Theorem 5.4. For every $x \geq 0$, we have

$$
\Theta(x)=Z(x) Y(x)^{-1},
$$

where the matrices $Y(x)$ and $Z(x)$ are given by

$$
\left(\begin{array}{c}
Z(x) \\
Y(x)
\end{array}\right)=e^{-C Q x}\left(\begin{array}{c}
-Q_{--}^{-1} Q_{-+} \\
I
\end{array}\right)=\left(\begin{array}{cc}
A(x) & B(x) \\
C(x) & D(x)
\end{array}\right)^{-1}\left(\begin{array}{c}
-Q_{--}^{-1} Q_{-+} \\
I
\end{array}\right) .
$$

Proof. Let us consider the following linear differential equation

$$
Y^{\prime}(x)=\left(-Q_{++}-Q_{+-} \Theta(x)\right) Y(x) \text { and } Y(0)=I .
$$

The function $\Theta(x)$ being continuous, this linear system has a unique solution which is invertible. We now define the $n_{-} \times n_{+}$matrix $Z(x)$ by

$$
Z(x)=\Theta(x) Y(x) .
$$

Using this definition, equation (14) becomes

$$
Y^{\prime}(x)=-Q_{++} Y(x)-Q_{+-} Z(x) .
$$

By differentiating $Z(x)$ with respect to $x$, we obtain from (12) and (15)

$$
\begin{aligned}
Z^{\prime}(x)= & \Theta^{\prime}(x) Y(x)+\Theta(x) Y^{\prime}(x) \\
= & \left(Q_{--} \Theta(x)+\Theta(x) Q_{++}+\Theta(x) Q_{+-} \Theta(x)+Q_{-+}\right) Y(x) \\
& +\Theta(x)\left(-Q_{++} Y(x)-Q_{+-} Z(x)\right) \\
= & Q_{--} Z(x)+\Theta(x) Q_{+-} \Theta(x) Y(x)+Q_{-+} Y(x)-\Theta(x) Q_{+-} Z(x) \\
= & Q_{--} Z(x)+Q_{-+} Y(x) .
\end{aligned}
$$


Putting together equations (15) and (16) we obtain

$$
\left(\begin{array}{c}
Z^{\prime}(x) \\
Y^{\prime}(x)
\end{array}\right)=\left(\begin{array}{cc}
Q_{--} & Q_{-+} \\
-Q_{+-} & -Q_{++}
\end{array}\right)\left(\begin{array}{c}
Z(x) \\
Y(x)
\end{array}\right)=-C Q\left(\begin{array}{c}
Z(x) \\
Y(x)
\end{array}\right),
$$

with $Y(0)=I$ and $Z(0)=-Q_{--}^{-1} Q_{-+}$. The solution to that equation is given by

$$
\left(\begin{array}{c}
Z(x) \\
Y(x)
\end{array}\right)=e^{-C Q x}\left(\begin{array}{c}
-Q_{--}^{-1} Q_{-+} \\
I
\end{array}\right)=\left(\begin{array}{cc}
A(x) & B(x) \\
C(x) & D(x)
\end{array}\right)^{-1}\left(\begin{array}{c}
-Q_{--}^{-1} Q_{-+} \\
I
\end{array}\right) .
$$

\section{$6 \quad$ Finite Buffer Case}

We suppose now that the fluid queue is of finite capacity and we denote that capacity by $x$. We are then interested to the evaluation of the probability, starting from a phase $i \in S^{+}$and a level $z$, to reach level $z$ in phase $j$ while staying above level $y$.

More formally, we introduce the $n_{+} \times n_{-}$matrix $K(x, y, z)$ whose entries are defined, for $i \in S^{+}, j \in S^{-}, x>0$ and $0 \leq z \leq y \leq x$, by

$$
K_{i, j}(x, y, z)=\operatorname{Pr}\left\{\varphi(\gamma(z))=j, H \leq y \mid \varphi(0)=i, X_{0}=z\right\},
$$

where $H$ denotes the minimum trough between instants 0 and $\gamma(z)$.

By the spatial homogeneity, we have

$$
K_{i, j}(x, y, z)=K_{i, j}(x-z, y-z, 0) .
$$

The $n_{+} \times n_{-}$matrix $\Gamma(x, z)$ whose entries are defined, for $i \in S^{+}, j \in S^{-}, x>0$ and $0 \leq z \leq x$, by

$$
\Gamma_{i, j}(x, z)=\operatorname{Pr}\left\{\varphi(\gamma(z))=j \mid \varphi(0)=i, X_{0}=z\right\}
$$

satisfies

$$
\Gamma(x, z)=K(x, x, z)=K(x-z, x-z, 0)=\Gamma(x-z, 0) .
$$

The $n_{+} \times n_{-}$matrix $\Gamma(x)$ whose entries are defined, for $i \in S^{+}, j \in S^{-}$and $x>0$, by

$$
\Gamma_{i, j}(x)=\operatorname{Pr}\left\{\varphi(\gamma(0))=j \mid \varphi(0)=i, X_{0}=0\right\},
$$

satisfies

$$
\Gamma(x)=\Gamma(x, 0) .
$$

A typical example of such paths is shown in Figure 3.

This situation is symmetric to the one shown in Figure 2. We denote by $k_{i, j}(x, y, z)$ the density associated with the distribution $K_{i, j}(x, y, z)$, i.e.

$$
k_{i, j}(x, y, z)=\frac{\partial K_{i, j}(x, y, z)}{\partial y} .
$$



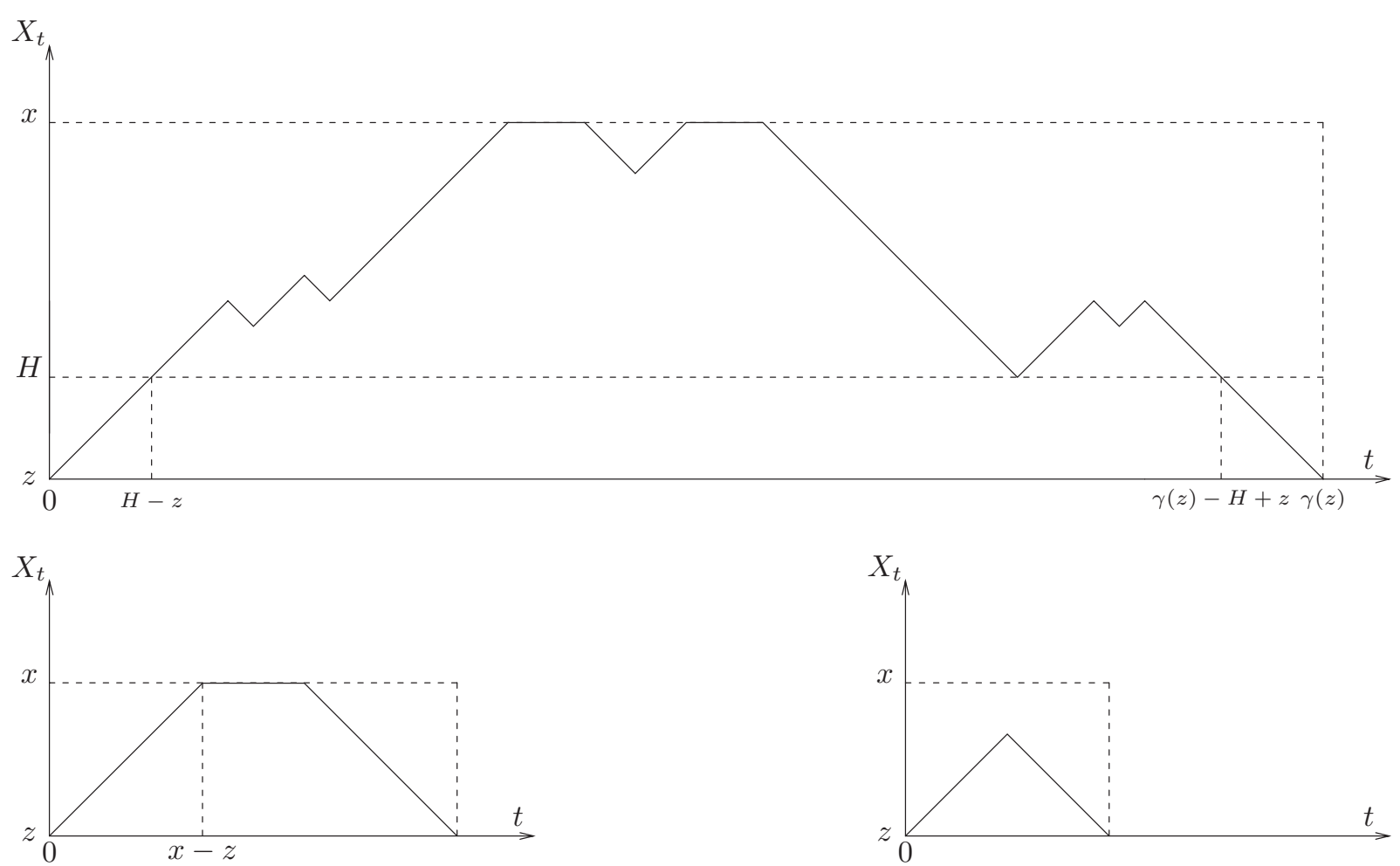

Figure 3: A period of level $>z$ in the finite buffer case.

Theorem 6.1. For $x>0$ and $0 \leq z \leq y \leq x$, we have

$$
K(x, 0, z)=\int_{z}^{x} e^{Q_{++}(y-z)} Q_{+-} e^{Q_{--}(y-z)} d y-Q_{++}^{-1} e^{Q_{++}(x-z)} Q_{+-} e^{Q_{--}(x-z)}
$$

and

$$
k(x, y, z)=e^{Q_{++}(y-z)} \Gamma(x-y) Q_{-+} \Gamma(x-y) e^{Q_{--}(y-z)} .
$$

Proof. The proof is quasi-identical to the proof of theorem 5.1.

By using the spatial homogeneity, the matrices $K(x, y, z)$ and $\Gamma(x)$ are given by the following equations.

For $x \geq 0$ and $0 \leq z \leq y \leq x$, we have

$$
K(x, y, z)=K(x, 0, z)+\int_{z}^{y} k(x, u, z) d u .
$$

For $x \geq 0$, we have $\Gamma(x)=\Gamma(x, 0)=K(x, x, 0)$, that is $\Gamma(x)=\int_{0}^{x} e^{Q_{++} y} Q_{+-} e^{Q_{--} y} d y-Q_{++}^{-1} e^{Q_{++} x} Q_{+-} e^{Q_{--} x}+\int_{0}^{x} e^{Q_{++} y} \Gamma(x-y) Q_{-+} \Gamma(x-y) e^{Q_{--} y} d y$.

This equation is the symmetric version of equation (11). We thus obtain, in the same way we got for equation (11), the matrix differential Riccati equation

$$
\Gamma^{\prime}(x)=Q_{++} \Gamma(x)+\Gamma(x) Q_{--}+\Gamma(x) Q_{-+} \Gamma(x)+Q_{+-} .
$$


This equation is identical to equation (4). The only difference concerns the initial condition. Here we have, from $(20), \Gamma(0)=-Q_{++}^{-1} Q_{+-}$. Following the same lines used in the proof of Theorem 4.4, we obtain $\Gamma(x)=Z(x) Y^{-1}(x)$, where

$$
\left(\begin{array}{c}
Y(x) \\
Z(x)
\end{array}\right)=e^{C Q x}\left(\begin{array}{c}
I \\
P_{+-}
\end{array}\right)
$$

and $P_{+-}=-Q_{++}^{-1} Q_{+-}$. Note that $P_{+-}$is a stochastic matrix. This can also be written as

$$
\left\{\begin{array}{l}
Y(x)=A(x)+B(x) P_{+-} \\
Z(x)=C(x)+D(x) P_{+-}
\end{array}\right.
$$

which gives

$$
\Gamma(x)=\left(C(x)+D(x) P_{+-}\right)\left(A(x)+B(x) P_{+-}\right)^{-1} .
$$

\section{Numerical Application}

In order to give a numerical example, we consider a fluid queue fed by 2 statistically independent and identical on-off sources. For each source, we assume that the on periods and the off periods form an alternating renewal process and their durations are exponentially distributed with mean $\lambda^{-1}=1$ and $\mu^{-1}=1$ respectively. The phase process $\varphi$ has thus 3 states 0,1 and 2 which count the number of on sources at each instant. To avoid the rescaling of the process discussed in Section 2, the input rates and the service rates of the fluid queue are supposed to be $r_{0}=r_{1}=-1$ and $r_{2}=1$. We then have $n_{-}=2$ and $n_{+}=1$ and the infinitesimal generator $Q$ and the matrix $C$ are given by

$$
Q=\left(\begin{array}{ccc}
-2 & 2 & 0 \\
1 & -2 & 1 \\
0 & 2 & -2
\end{array}\right) \quad \text { and } \quad C=\left(\begin{array}{ccc}
-1 & 0 & 0 \\
0 & -1 & 0 \\
0 & 0 & 1
\end{array}\right)
$$

The stationary distribution of process $\varphi$ is given by $\pi=(1 / 4,1 / 2,1 / 4)$ and the stability condition $-\pi_{0}-\pi_{1}+\pi_{2}<0$ is satisfied. Calculating the matrix $e^{C Q x}$ gives us

$$
\begin{gathered}
A(x)=\left(\begin{array}{cc}
\frac{1}{2}+\frac{5+3 \sqrt{5}}{20} e^{(1+\sqrt{5}) x}+\frac{5-3 \sqrt{5}}{20} e^{(1-\sqrt{5}) x} & 1-\frac{5+\sqrt{5}}{10} e^{(1+\sqrt{5}) x}-\frac{5-\sqrt{5}}{10} e^{(1-\sqrt{5}) x} \\
\frac{1}{2}-\frac{5+\sqrt{5}}{20} e^{(1+\sqrt{5}) x}-\frac{5-\sqrt{5}}{20} e^{(1-\sqrt{5}) x} & 1+\frac{\sqrt{5}}{5} e^{(1+\sqrt{5}) x}-\frac{\sqrt{5}}{5} e^{(1-\sqrt{5}) x}
\end{array}\right), \\
C(x)=\left(\begin{array}{cc}
\frac{1}{2}-\frac{5-\sqrt{5}}{20} e^{(1+\sqrt{5}) x}-\frac{5+\sqrt{5}}{20} e^{(1-\sqrt{5}) x} & 1-\frac{5-3 \sqrt{5}}{10} e^{(1+\sqrt{5}) x}-\frac{5+3 \sqrt{5}}{10} e^{(1-\sqrt{5}) x}
\end{array}\right), \\
B(x)=\left(\begin{array}{c}
-\frac{1}{2}+\frac{5-\sqrt{5}}{20} e^{(1+\sqrt{5}) x}+\frac{5+\sqrt{5}}{20} e^{(1-\sqrt{5}) x} \\
-\frac{1}{2}+\frac{5-3 \sqrt{5}}{20} e^{(1+\sqrt{5}) x}+\frac{5+3 \sqrt{5}}{20} e^{(1-\sqrt{5}) x}
\end{array}\right), \\
D(x)=-\frac{1}{2}+\frac{15-7 \sqrt{5}}{20} e^{(1+\sqrt{5}) x}+\frac{15+7 \sqrt{5}}{20} e^{(1-\sqrt{5}) x} .
\end{gathered}
$$


We then have

$$
\operatorname{det}(A(x))=\frac{15+7 \sqrt{5}}{20} e^{(1+\sqrt{5}) x}-\frac{1}{2} e^{2 x}+\frac{15-7 \sqrt{5}}{20} e^{(1-\sqrt{5}) x}
$$

and

$$
A^{-1}(x)=\frac{1}{\operatorname{det}(A(x))} M(x)
$$

where

$$
M(x)=\left(\begin{array}{cc}
1+\frac{\sqrt{5}}{5} e^{(1+\sqrt{5}) x}-\frac{\sqrt{5}}{5} e^{(1-\sqrt{5}) x} & -1+\frac{5+\sqrt{5}}{10} e^{(1+\sqrt{5}) x}+\frac{5-\sqrt{5}}{10} e^{(1-\sqrt{5}) x} \\
-\frac{1}{2}+\frac{5+\sqrt{5}}{20} e^{(1+\sqrt{5}) x}-\frac{5-\sqrt{5}}{20} e^{(1-\sqrt{5}) x} & \frac{1}{2}+\frac{5+3 \sqrt{5}}{20} e^{(1+\sqrt{5}) x}+\frac{5-3 \sqrt{5}}{20} e^{(1-\sqrt{5}) x}
\end{array}\right) .
$$

The matrix $\Psi(x)=\left(\Psi_{2,0}(x), \Psi_{2,1}(x)\right)$ is given, from Theorem 4.4, by $\Psi(x)=C(x) A^{-1}(x)$, which gives

$$
\Psi_{2,0}(x)=\frac{(5+\sqrt{5}) e^{(1+\sqrt{5}) x}-10 e^{2 x}+(5-\sqrt{5}) e^{(1-\sqrt{5}) x}}{(15+7 \sqrt{5}) e^{(1+\sqrt{5}) x}-10 e^{2 x}+(15-7 \sqrt{5}) e^{(1-\sqrt{5}) x}},
$$

and

$$
\Psi_{2,1}(x)=\frac{(10+6 \sqrt{5}) e^{(1+\sqrt{5}) x}-20 e^{2 x}+(10-6 \sqrt{5}) e^{(1-\sqrt{5}) x}}{(15+7 \sqrt{5}) e^{(1+\sqrt{5}) x}-10 e^{2 x}+(15-7 \sqrt{5}) e^{(1-\sqrt{5}) x}} .
$$

By taking the limit when $x$ tends to infinity, we get, as expected

$$
\Psi=(-2+\sqrt{5}, 3-\sqrt{5}) .
$$

By adding $\Psi_{2,0}$ and $\Psi_{2,1}(x)$, we obtain the distribution of the maximum level $M$ during a busy period, i.e.

$$
\operatorname{Pr}\{M \leq x\}=\frac{(15+7 \sqrt{5}) e^{(1+\sqrt{5}) x}-30 e^{2 x}+(15-7 \sqrt{5}) e^{(1-\sqrt{5}) x}}{(15+7 \sqrt{5}) e^{(1+\sqrt{5}) x}-10 e^{2 x}+(15-7 \sqrt{5}) e^{(1-\sqrt{5}) x}}
$$

Suppose now that the buffer is of finite capacity $x$. The matrix $\Gamma(x)=\left(\Gamma_{2,0}(x), \Gamma_{2,1}(x)\right)$ is given, from relation (22), by

$$
\Gamma(x)=\left(C(x)+D(x) P_{+-}\right)\left(A(x)+B(x) P_{+-}\right)^{-1},
$$

which gives, since $P_{+-}=\left(\begin{array}{ll}0 & 1\end{array}\right)$,

$$
\Gamma_{2,0}(x)=\frac{(5-\sqrt{5})\left(e^{(1+\sqrt{5}) x}-e^{(1-\sqrt{5}) x}\right)}{(5+3 \sqrt{5}) e^{(1+\sqrt{5}) x}-(15-7 \sqrt{5}) e^{(1-\sqrt{5}) x}},
$$

and

$$
\Gamma_{2,1}(x)=\frac{4 \sqrt{5} e^{(1+\sqrt{5}) x}-(10-6 \sqrt{5}) e^{(1-\sqrt{5}) x}}{(5+3 \sqrt{5}) e^{(1+\sqrt{5}) x}-(15-7 \sqrt{5}) e^{(1-\sqrt{5}) x}} .
$$

Again, as expected, we have

$$
\lim _{x \longrightarrow \infty} \Gamma(x)=\Psi .
$$




\section{References}

[1] S. Ahn and V. Ramaswami. Fluid flow models and queues - a connection by stochastic coupling. Comm. Statist. Stochastic Models, 19(3):325-348, 2003.

[2] D. Anick, D. Mitra, and M. M. Sondhi. Stochastic theory of a data-handling system with multiple sources. Bell System Tech. J., 61(8):1871-1894, 1982.

[3] S. Asmussen. Stationary distributions for fluid flow models with or without brownian noise. Stochastic Models, 11(1):21-49, 1995.

[4] A. Badescu, L. Breuer, A. da Silva Soares, G. Latouche, M.-A. Remiche, and D. Stanford. Risk processes analyzed as fluid queues. Scandinavian Actuarial Journal, 2:127-141, 2005.

[5] N. Barbot and B. Sericola. Stationary solution to the fluid queue fed by an M/M/1 queue. Journ. Appl. Probab., 39:359-369, 2002.

[6] N. G. Bean, M. M. O'Reilly, and P. G. Taylor. Algorithms for the first return probabilitiesfor stochastic fluid flows. Stochastic Models, 21(1), 2005.

[7] N. G. Bean, M. M. O'Reilly, and P. G. Taylor. Hitting probabilities and hitting times for stochastic fluid flows. Stochastic Processes and their Applications, 115:1530-1556, 2005.

[8] A. da Silva Soares and G. Latouche. Further results on the similarity between fluid queues and QBDs. In G. Latouche and P. Taylor, editors, Proc. of the 4th Int. Conf. on MatrixAnalytic Methods, pages 89-106, Adelaide, 2002. World Scientific.

[9] A. da Silva Soares and G. Latouche. Matrix-analytic methods for fluid queues with finite buffers. Performance Evaluation, 63(4):295-314, 2006.

[10] F. Guillemin, G. Rubino, B. Sericola, and A. Simonian. Transient analysis of statistical multiplexing of data on an ATM link. In V. Ramaswami and P. E. Wirth, editors, Proceedings of the 15th International Teletraffic Congress : Teletraffic Contribution for the Information Age (ITC'15), Washington D.C., USA, 1997. Elsevier.

[11] F. Guillemin and B. Sericola. Stationary analysis of a fluid queue driven by some countable state space Markov chain. Methodology and Computing in Applied Probability (to appear), 2007.

[12] L. Kosten. Stochastic theory of data-handling systems with groups of multiple sources. In Proceedings of the IFIP WG 7.3/TC 6 Second International Symposium on the Performance of Computer-Communication Systems, Zurich, Switzerland, pages 321-331, 1984.

[13] D. Mitra. Stochastic theory of a fluid model of producers and consumers coupled by a buffer. Advances in Applied Probability, 20:646-676, 1988.

[14] V. Ramaswami. Matrix analytic methods for stochastic fluid flows. In D. Smith and P. Hey, editors, Proceedings of the 16th International Teletraffic Congress : Teletraffic Engineering in a Competitive World (ITC'16), pages 1019-1030, Edinburgh, UK, 1999. Elsevier.

[15] M.-A. Remiche. Compliance of the token-bucket model with markovian traffic. Stochastic Models, 21:615-630, 2005.

PI $n^{\circ} 1832$ 
[16] L. C. G. Rogers. Fluid models in queueing theory and wiener-hopf factorization of markov chains. Annals of Applied Probability, 4(2):390-413, 1994.

[17] G. Rubino and B. Sericola. Accumulated reward over the $\mathrm{n}$ first operational periods in faul-tolerant computing systems. Technical Report 1028, INRIA, 1989.

[18] B. Sericola. Occupation times in Markov processes. Comm. Statist. Stochastic Models, 16(5), 2000.

[19] B. Sericola. A finite buffer fluid queue driven by a Markovian queue. Queueing Systems Theory and Applications, 38:213-220, 2001.

[20] E.A. van Dorn and W.R. Scheinhardt. A fluid queue driven by an infinite-state birth and death process. In V. Ramaswami and P. Wirth, editors, Proc. ITC 15, pages 465-475, Amsterdam, 1997. Elsevier. 